\title{
Politische Kommunikation: lehrreicher Vergleich der Wahlkämpfe in den USA und Deutschland
}

Burgard, Jan Philipp: Von Obama siegen lernen oder „Yes, We Gähn!'? Der Jahrhundertwahlkampf und die Lehren für die politische Kommunikation in Deutschland, Nomos Verlagsgesellschaft, Baden-Baden 2011, 256 Seiten, € 49,-.

Vergleicht man Äpfel mit Birnen, wenn man Gemeinsamkeiten und Unterschiede zwischen dem Präsidentschaftswahlkampf zwischen Barack Obama und John McCain im Jahre 2008 und dem Wahlkampf im Vorfeld der Bundestagswahl 2009 zu ermitteln sucht? Wenngleich politische Systeme und Kommunikationskultur in Deutschland und den USA verschieden sind, erscheint der Forschungsansatz Jan Philipp Burgards in seiner bei Eckhard Jesse an der TU Chemnitz entstandenen politikwissenschaftlichen Dissertation aus dem Jahre 2011 bestechend: Im Fokus steht die Frage, ob und auf welche Weise der Wahlkampf in den USA 2008 denjenigen in Deutschland 2009 beeinflusst hat.

Burgard skizziert nach einer kurzen Einführung die politischen Kommunikationsbedingungen in beiden Ländern und verdeutlicht die für Wahlkämpfe nicht folgenlosen Unterschiede etwa im Hinblick auf die Rolle von Präsident beziehungsweise Bundeskanzler, die Parteiverankerung von Kandidaten und die Fraktionsdisziplin, das Wahlsystem, die Parteien- und Wahlkampffinanzierung sowie die Bedeutung und Organisation der Medien. In einem geschichtlichen Rückblick schildert Burgard vor allem die im technischen Fortschritt gründende Genese der politischen Kommunikation in den USA und benennt wesentliche Charakteristika der Wahlkämpfe: starke Personalisierung, TV-orientierte Kampagnenführung, in jüngerer Zeit unter Nutzung des Internets, direkte Wähleransprache, Auslagerung der Wahlkampfführung aus den Parteien, intensive Meinungsforschung, „Fundraising “ für den Wahlkampf und das so genannte „Negative Campaining“ - die bewusste Verunglimpfung des politischen Kontrahenten. Dem stellt Burgard die deutsche Geschichte gegenüber und zeigt, dass schon Konrad Adenauer im Wahlkampf 1953 auf eine am US-amerikanischen Vorbild orientierte Personalisierung setzte. Entsprechende Merkmale zeigten sich auch 1961 bei der Nominierung Willy Brandts, beim „Telekanzler“ Helmut Schmidt und selbst beim mit „defizitärem Mediengeschick“ versehenen Helmut Kohl.

Besondere Hervorhebung erfahren die „Inszenierungen“ der Kandidaten. Detailreich werden die Kampagnen von Obama und McCain behandelt, vor allem die Art und Weise der Einführung ihrer Biographien. Auch die jeweiligen „Messages“ werden hinsichtlich ihrer Wahlkampfeignung kritisch beleuchtet, der Verlauf der Nominierungsparteitage und des Vorwahlkampfes beschrieben. Instruktiv ist die Untersuchung, wie sich der US-Wahlkampf auf den Wettbewerb zwischen Angela Merkel und Frank-Walter Steinmeier ausgewirkt hat. Beide Lebensläufe und politische Karrieren werden ausführlich nachgezeichnet. Burgard verdeutlicht, dass sich namentlich der Wahlkampf Merkels stark an dem Obamas orientiert hat - dies belegt er vor allem am Beispiel von Fernsehwerbespots der Union, in denen die Biographie der Amtsinhaberin in den Vordergrund gestellt und sie als „überparteilich“ inszeniert worden sei. Parallel zu den Darlegungen über die USA finden auch die Botschaften der deutschen Kampagnen und die Parteitage kritische Berücksichtigung. Ein abschließender Vergleich der Inszenierung der Kandidaten ergibt, dass der deutsche Wahlkampf der starken Tendenz zur Personalisierung in den USA gefolgt sei. Die gewisse „programmatische Leere“ des Bundestagswahlkampfs 2009 hatte jedoch, wie Burgard zeigt, kein 
unmittelbares Vorbild im US-Wahlkampf von 2008, denn dort wurden zahlreiche (vor allem außenpolitische und ökonomische) Sachthemen ins Blickfeld gerückt. Er betont, dass es Obama letztlich besser gelungen sei, seine Programmatik verständlich zu kommunizieren und mit seiner Biographie in glaubhafte Kongruenz zu bringen. Wiederum „spiegelbildlich" behandelt er sodann die Wahlkampfthemen in Deutschland und spricht sich angesichts der seiner Auffassung nach von den Parteien gewollten „Entsachlichung" des Wahlkampfs von 2009 für eine Intensivierung des thematischen „Agenda Settings“ der deutschen Parteien aus.

Sodann beschreibt Burgard den Wechsel Obamas von der traditionellen „Battleground“Strategie (der Konzentration auf „sichere“ Staaten im Wahlkampf) zur „50-Staaten-Strategie" (einem landesweiten Wahlkampf), zeitnahe Wählermobilisierungsaktionen und die erhebliche Bedeutung der Wahlkampfstrategen. Damit vergleicht er die Wahlkampfmanager von CDU und SPD, denen er konzeptionelle Schwächen vorhält, sowie die strategischen Grundlinien des Wahlkampfs von 2009. Jeweils eigene Kapitel bilden die Frage der Einbeziehung der Medien in den Wahlkampf und die Nutzung des Internets als „Wunderwaffe“. Burgard legt die Medienstrategien der Kandidaten einschließlich des (in Deutschland nur marginal auszumachenden) „Negative Campaignings“, aber auch die Unterschiede in der Berichterstattung dar. Im Hinblick auf die Nutzung des Internets untersucht er wiederum vergleichend die Partei-Homepages (einschließlich ihrer interaktiven Elemente und OnlineVideos der Kandidaten), die Eignung des Netzes als Organisations- oder Fundraising-Instrument, die Nutzung externer Netzwerke (zum Beispiel Facebook) und die Bedeutung von Blogs. Sehr interessant sind dabei vor allem auch die Ausführungen über das „Microtargeting" via Internet, das gezielte Identifizieren und Ansprechen von Wählergruppen.

In der Schlussbetrachtung wertet Burgard die Inszenierung Obamas als „virtuos“ und weist auf die Vorbildfunktion für deutsche Politiker hin. Zwar sei die Übernahme von etwas "Glamour" aus den USA in den Bundestagswahlkampf 2009 nur teilweise geglückt, bei Angela Merkel sei aber insbesondere im Vergleich mit 2005 ein deutlicher Lernerfolg erkennbar. Er regt an, die Kandidatenfindung in den Parteien zu überdenken - auch, um das Fortschreiten der „Politikverdrossenheit“ in der Bevölkerung zum Stillstand zu bringen und dem „demokratischen Prozess neues Leben einzuhauchen“ (S. 231). Erforderlich seien mitreißende, rhetorisch befähigte Politiker mit klaren inhaltlichen Agenden. Wünschenswert seien eine stärkere „Durchlässigkeit“ von Politik, Wissenschaft, Wirtschaft und Medien sowie eine Intensivierung der Nutzung des Internets zur Mobilisierung und Koordination der Wähler.

Es mag der juristischen Prägung des Rezensenten geschuldet sein, dass er sich bei der Lektüre der im Übrigen vorzüglichen Dissertation von Jan Philipp Burgard insgesamt ein Mehr an wissenschaftlicher Kontroverse gewünscht hätte. Auch die im Untertitel angekündigten „Lehren“ für die politische Kommunikation in Deutschland hätten etwas prägnanter herausgearbeitet werden können. Dies schmälert keineswegs die Verdienste des Autors, der eine detailreiche, analytisch tiefgehende, anregende und gut lesbare Arbeit vorgelegt hat. Die darstellerische Stringenz und das weitestgehende Fehlen ausufernder Darlegungen zu politikwissenschaftlichen Streitfragen werden wohl sogar zu einer - sehr zu wünschenden - breiten Rezeption der Schrift beitragen, die vor allem Politikern jeglicher Couleur und ihren Wahlkampfexperten als lehrreiche Fundgrube für Anregungen zu einer personalisierten Kandidatenpräsentation ans Herz gelegt werden kann. 